\section{Thieme journals fulfill your need for contemporary resources}

A diverse group of award-winning Editors complement our journals in a vast variety of specialties.

\title{
Synergistic activity of cold pressed seed oils from Indian neem (Azadirachta indica), karanja (Pongamia glabra) and aloe vera gel (Aloe barbadensis) in a cosmeceutical moisturizer
}

RF Oliveira $\underline{1}, \underline{2}, \underline{3}, \underline{8}$, L Barreiro $\underline{\mathbf{2}}, \underline{4}$, A Cruz $\underline{2}$, AI Oliveira $\underline{2}, \underline{\mathbf{5}}$, A Dias $\underline{\mathbf{5}}$, C Pinho $\underline{\mathbf{2}}, \underline{\mathbf{5}}$, S Fernandes $\underline{2}, \underline{\mathbf{5}}$, NM Santos $\underline{\mathbf{7}}, \underline{\mathbf{8}}$, J Morgado $\underline{\mathbf{1}}, \underline{\mathbf{8}}$

${ }^{1}$ M\&M Biotechnology, Porto, Portugal

${ }^{2}$ Núcleo de Investigação e Intervenção em Farmácia (NIIF), Centro de Investigação em Saúde e Ambiente (CISA), Escola Superior de Tecnologia da Saúde do Porto (ESTSP), Instituto Politécnico do Porto (IPP), Vila Nova de Gaia, Portugal

(ESTSP), Instituto Politécnico do Porto (IPP), Vila Nova de Gaia, Portugal
${ }^{3}$ Health Sciences Autonomous Section, University of Aveiro, Aveiro, Portugal

3 Health Sciences Autonomous Section, University of Aveiro, Aveiro, Portugal
4REQIMTE, Departamento de Ciências Químicas, Faculdade de Farmácia, Universidade do Porto, Porto, Portugal

${ }^{5}$ CITAB-UM, AgroBioplant group, Department of Biology, University of Minho, Braga, Portugal

${ }^{6}$ Ciências Químicas e das Biomoléculas, Escola Superior de Tecnologia de Saúde do Porto (ESTSP), Instituto Politécnico do Porto (IPP), Vila Nova de Gaia, Portugal

${ }^{7}$ Chemical Wood Technology, University of Hamburg, Germany

8 M\&M - GluckNeem Develop Team, Porto, Portugal

\section{Congress Abstract}

The increased interest and knowledge on the beneficial activity of plants and their compounds in human health have contributed to the development of several drugs, widely used in clinical practice worldwide. Over the last years the incorporation of natural products, particularly plant based products, in cosmeceuticals has also increased, leading the industry to adapt and redirect its production in order to correspond to the consumers' needs. Scientific studies are, however, still insufficient leading to the constant introduction in the market of products with unknown benefits and risks. Azadirachta indica (neem) and Pongamia glabra (karanja) oils, and Aloe barbadensis (aloe vera gel) are three components that have been employed in topical cosmeceutical products. On the present study the optimization of a pharmaceutical preparation originated a cream with extracts of the three plants mentioned above. This formulation was then applied in adult volunteers (more then 150) with skin disorders, namely psoriases and skin burns. The synergy of the properties of these plants, in specific treatment situations, resulted in very positive results. In all cases, after repeated applications, notorious improvements or complete treatment were observed, without side effects. Examples of the treatments are shown in Figure 1. The three plants revealed to be associated to a high degree of skin hydration, contributing, therefore, to cellular skin regeneration. Ongoing clinical evidences have shown that mixtures with these three plants are effective in the treatment of some skin injuries. The results demonstrate a similar effectiveness, when comparing to the conventional treatments.

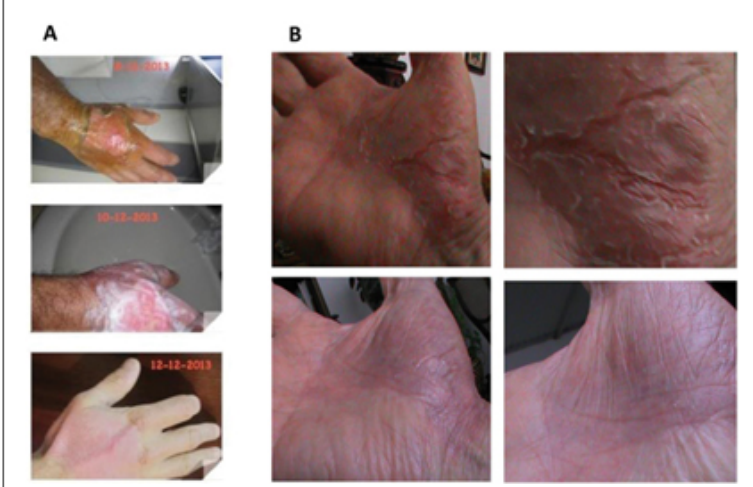

Fig. 1: Treatment of a hand-burn in 96h (column A) and of a palmoplanar psoriasis in 3 months (column B).

Keywords: Azadirachta indica, Pongamia glabra, Aloe barbadensis, dermocosmetics, psoriases, skin burns 